\title{
Effects of maze geometry and experience on exploratory behavior in the rat
}

\author{
H. J. USTER, K. BÄTTIG, and H. H. NÄGELI \\ Swiss Federal Institute of Technology, Department of Behavioral Sciences, Zurich, Switzerland
}

\begin{abstract}
Rats were exposed twice in a rotated sequence to a series of six mazes, consisting of hexagonal alleys, balanced for different alley length and structural complexity. Locomotor activity increased with alley length and decreased with structural complexity of the mazes. Locomotion became less stereotyped with increased experience, showing an increasing number of turns, less constant velocity, loss of the initial preference for outward leading alleys and weakening of the forward tendency at reentry from side alleys into hexagonal alleys. In contrast to these qualitative changes of locomotion, the amount of activity remained almost unchanged throughout the experiment. The results suggest that these increases in locomotion complexity depend upon complex interactions between experience and stimulus content of the mazes.
\end{abstract}

The degree of complexity and the degree of novelty of an exploratory situation are the main determinants of its incentive value (Berlyne \& Slater, 1951; Dember \& Earl, 1957; Dutch \& Brown, 1971; Taylor, 1971). Following Montgomery's initial research (Montgomery, 1952), many suggested that alternation behavior is an expression of a rat's tendency to optimize variation of information input. However, little attention has been directed to the rat's exploratory behavior in complex mazes. Glanzer (1971) showed that a complex maze was explored successively further and further from the starting point during repeated exposure. In line with Glanzer's findings and his probability model for linear movement, a study by Bättig \& Wanner (1967), using a combined maze and open field situation, showed that initial activity over a repeated number of sessions remained constant, that the decrease in intra-session activity declined as a function of time, and that rats demonstrated a changing preference for different individual places in the maze during one exposure.

Fowler (1967) questioned the validity of using a single measure of maze exploration. Usually the sole dependent variable measured is rate of maze unit traversal. Quoting Eisenberger (1972), "Exploratory behavior in the maze might be reflected in two competing exploratory tendencies: (1) to explore more maze sections per unit time and (2) to examine individual maze sections in more detail." In the present study the application of a photocell method for continuous recording of locomotion (Bättig \& Rhiner, 1966) made it possible to include behavioral variables besides activity and intrasession activity decrease. These included the number of turns, decision frequencies at maze branchings, and photocell to photocell transition times at distinct maze locations. Furthermore, the maze used by Bättig \& Wanner (1967) was intersectioned so as to obtain six mazes consisting essentially of hexagonal alleys with and without side

This study was supported by Grant No. 3.1000 .73 from the Swiss National Foundation for Scientific Research. Requests for reprints should be sent to Prof. K. Battig, Department of Behavioral Sciences, Swiss Federal Institute of Technology, Turnerstrasse 1, Zurich, Switzerland. alleys and which were balanced for area (alley length), structural complexity, and side alleys leading toward the periphery or toward the center. The animals were exposed in a rotated sequence to these mazes in order to test for the effects of maze geometry. Furthermore, this sequence was repeated once in order to evaluate possible effects of experience.

\section{METHOD}

\section{Subjects}

The experiments were carried out with 36 male, naive Wistar rats (breeding colony CIBA-GEIGY, Basel), weighing from $300-350 \mathrm{~g}$. The animals were housed in groups (6 66 animals), in conventional cages, in the room in which they were tested and which was used for no other purposes throughout the experiment. Food and water were available ad lib. The experimental room was illuminated by fluorescent light from $6 \mathrm{a} . \mathrm{m}$. to 6 p. m. and with a dim red light from 6 p. m. to 6 a. m.

\section{Apparatus}

Six alley configurations were obtained by intersectioning a complex hexagonal maze (Bättig \& Wanner, 1967; Bättig, 1969), with barriers. Figure 1 shows the complete maze and the resulting arrangements of different area and complexity. The area value of the mazes is given by the number of area units and the complexity value by the number of corners and radial alleys. The wooden maze was covered with a black plastic cloth. The alleys,

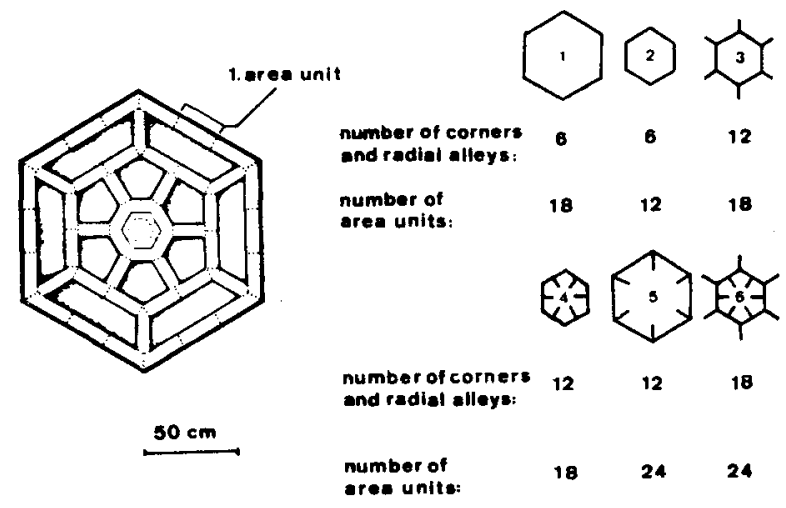

Figure 1. Left: ground plan of the complete hexagonal maze. Right: the six experimental alley configurations. 
Table 1

The Effects of Maze Area and Complexity on Activity Variables and Number of Turns

\begin{tabular}{|c|c|c|c|c|c|c|c|c|c|c|c|c|c|}
\hline \multirow[b]{2}{*}{ Mazes } & \multicolumn{6}{|c|}{ (a) Equal Complexity } & \multicolumn{7}{|c|}{ (b) Equal Area } \\
\hline & 2 & & 1 & 3 & & 5 & 1 & 3 & 1 & 4 & 5 & & 6 \\
\hline Number of area units & 12 & & 18 & 18 & & 24 & 18 & 18 & 18 & 18 & 24 & & 24 \\
\hline Number of corners and radial alleys & 6 & & 6 & 12 & & 12 & 6 & 12 & 6 & 12 & 12 & & 18 \\
\hline Average activity per $30 \mathrm{sec}$ & 16.7 & ** & 19.4 & 16.1 & $* *$ & 19.4 & 19.4 & $* * \quad 16.1$ & $19.4 * * 1$ & 15.2 & 19.4 & $* * 1$ & 4.8 \\
\hline $\begin{array}{l}\text { Linearized intrasession activity } \\
\text { decrease per } 30 \mathrm{sec}\end{array}$ & 1.39 & & 1.37 & 1.18 & $* *$ & 1.35 & 1.37 & ** 1.18 & $1.37 * *$ & 1.07 & 1.35 & $* *$ & .79 \\
\hline $\begin{array}{l}\text { Increase of turns from first to } \\
\text { second experimental block }\end{array}$ & 5.8 & & 5.9 & 4.6 & & 3.2 & 5.9 & 4.6 & $5.9 *$ & 3.4 & 3.2 & & 2.4 \\
\hline
\end{tabular}

${ }^{* *} p<.01$

$8 \mathrm{~cm}$ wide and $15 \mathrm{~cm}$ deep, were painted uniformly black. In each of the 42 area units, a weakly illuminated lamp was fitted opposite to a photosensitive cell. A rat passing through the light beam was registered continuously on a punch tape. Separate codes were used for each of the 42 area units. A code was punched into the tape at $0.2-\mathrm{sec}$ intervals, by means of an electronic device (Bättig \& Rhiner, 1966). Direct observation of the locomotor behavior was made possible by $\mathbf{4 2}$ monitor lamps, coupled with the photoresistors and mounted adequately on a checkboard. A monotonous and weak background noise was produced by the puncher, installed in a sound-attenuating enclosure.

\section{Procedure and experimental design}

All experiments were carried out at night between $9 \mathrm{p.m}$. and $5 \mathrm{a}$. $\mathrm{m}$. At the start of each run, an animal was placed into a definite area unit of the middle or outer hexagonal alley. Six minutes later the animal was removed from the maze, and the maze floor was wiped with a wet sponge. On the day preceding the start of the experiment, each rat was given a habituation run of $2 \mathrm{~min}$ in the complete (i.e., not intersectioned) maze. Each of the 6 groups consisted of 6 animals randomly selected. The 6 groups were exposed once a day on 6 successive days to the 6 mazes, following the latin square method in the reversed order of the mazes, given in Figure 1. After a 2-day break, this testing program was replicated. On each experimental day the sequence of the groups, as well as the sequence of the individual animals within each group, was rotated in a balanced order.

\section{Data processing and statistics}

The data on punchtape were processed by a CDC 6000 computer in order to obtain the desired variables, such as activity (number of photocell activations), linearized intrasession activity decrease (regression analysis), turns (consecutive interruptions of three neighboring light beams repeated in reversed order), frequency of entering radial alleys at the branchings (computed separately for outward and inward leading radial alleys), transition times from one to a neighboring photocell calculated separately for distinct maze locations, and the frequency of changing (as opposed to continuing) running direction upon leaving a radial alley and reentering the hexagonal alley. Separate analyses of variance were calculated for each of these behavioral variables in order to evaluate the effects of the 6 mazes, the 2 experimental blocks, and the 12 testing days. Significance between mean values was ascertained with the
Duncan test at the $\mathrm{p}<0.05\left(^{*}\right)$ and at the $\mathrm{p}<0.01\left(^{* *}\right)$ level. Locomotion patterns of a few typical runs were additionally plotted with a special computer program.

\section{RESULTS}

(a) The effects of area and structural complexity on activity-related measures and the number of turns

The geometry of the testing arrangement allowed two comparisons between mazes differing with respect to area only ( 1 vs. 2 and 3 vs. 5) and three comparisons between mazes differing with respect to structural complexity only ( 1 vs. 3,1 vs. 4 , and 5 vs. 6 ). These maze characteristics appeared to influence activity, intrasession activity decrease, and the average increase in number of turns from the first to the second experimental block, as shown in Table 1. Activity increased with area and decreased with complexity of the mazes, the respective differences being highly significant in all five comparisons. Intrasession habituation, as represented by the linearized, average intrasession activity decrease, followed a more or less similar course. The decrease of this variable with increasing complexity was significant in all three comparisons. However, an analogous increase of this variable with increasing area was found in only one of the two respective comparisons. These intermaze differences in activity and intrasession activity decrease were also found to be of the same order if calculated separately for the first and second experimental blocks. The number of turns failed to show a direct relationship with maze area and complexity. However, a different picture was given by the comparison of the number of turns calculated separately for the two experimental blocks. The more complex mazes yielded a relatively high number of turns during the first experimental block, and only a modest increase occurred in the second block. The opposite was true for the less complex mazes, which yielded a low number of

Table 2

Frequency of Entering Radial Alleys

\begin{tabular}{lllllcc}
\hline Mazes & 3 & 4 & 3 & 5 & 6 & 6 \\
Angle (Deg) & 120 & 90 & 120 & 60 & 90 & 120 \\
Entering & Inward & Outward & Inward & Outward & Inward & Outward \\
Frequencies (Percentage) & $17.78 * *$ & 28.51 & $19.91 * *$ & 28.51 & $17.75 \quad * *$ & 27.24 \\
\hline
\end{tabular}

${ }^{* *} p<.01$ 
turns during the first experimental block and a relatively strong subsequent increase. This differential tendency, represented in the table as the increase of turns from the 1 st to the 2nd block, was present in all three comparisons for complexity but reached significance in only one of them. Furthermore, this measure failed to show any relation to maze area.

\section{(b) Choice behavior at radial alley branchings}

Per cent frequencies of entering radial alleys at passing branchings are compared separately in Table 2 for the alleys leading outward and inward. The outward choice frequencies were significantly higher than the inward choice frequencies without any consistent relation to the angles of the branchings, within the scope of the possible comparisons.

\section{(c) Transition times at distinct maze locations}

The average photocell-to-photocell transition times have been grouped for comparable maze locations in Table 3. These transition times were always shorter for straight than for corner sections, with the exception of Mazes 4 and 6, where this trend was small and nonsignificant or even reversed. In these two mazes the straight sections contained branchings which appear to have slowed the subjects in favor of an increased speed at the corners, regardless of whether these corners confronted the animals with a side alley choice or not. The longest transition times were found at leaving radial alleys where the animal had to decide on the direction to be taken at reentering the hexagonal alley.

\section{(d) The effect of experience on the behavioral measures}

As seen in the two graphs of locomotion patterns, which are typical for the behavior of the rats in this experiment, obvious qualitative changes in locomotor behavior did take place over the 12 testing days (Figure 2). Plot a represents the first run of a rat in Maze 1 and Plot $b$ shows the behavior of the same rat in the same maze after the experience of the first experi-

Table 3

Transition Times in Seconds at Distinct Maze Locations

\begin{tabular}{|c|c|c|c|c|}
\hline \multirow{3}{*}{$\begin{array}{c}\text { Maze } \\
\text { Number }\end{array}$} & \multicolumn{2}{|c|}{$\begin{array}{c}\text { Transition Times (sec) } \\
\text { Along Hexagonal Alley } \\
\text { or Into Radial } \\
\text { Alleys at }\end{array}$} & \multicolumn{2}{|c|}{$\begin{array}{c}\text { Transition Times (sec) } \\
\text { at Reentry of Hexa- } \\
\text { gonal Alley and } \\
\text { After Leaving }\end{array}$} \\
\hline & \multicolumn{2}{|c|}{ Sections } & \multicolumn{2}{|c|}{ Radial Alley } \\
\hline & Straight & Corner & Outer & Inner \\
\hline 1 & .75 & 1.80 & & \\
\hline 2 & 1.10 & 1.45 & & \\
\hline 3 & .75 & 1.40 & 3.10 & \\
\hline 4 & 1.40 & 1.15 & & 3.70 \\
\hline 5 & .60 & 1.85 & 3.25 & \\
\hline 6 & 1.10 & 1.20 & 2.55 & 4.05 \\
\hline
\end{tabular}

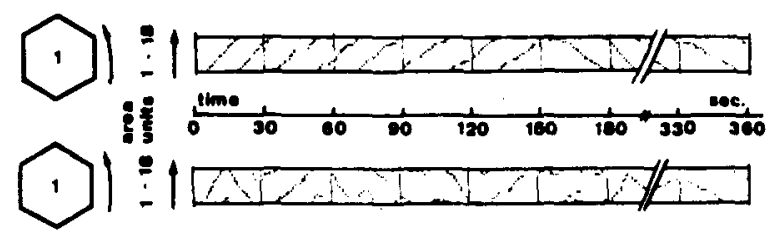

Figure 2. Locomotion plot of an individual rat in Maze 1 during the first (a) and the second exposure (b). Between the two exposures the animal experienced the other five mazes. The abscissa represents time; the ordinate refers to the 18 area units of the maze.

mental block, in which the rat was given runs in all of the other five mazes. The first run was characterized by a stereotyped pacing off with only one turn and a relatively constant velocity. After 6 days, the run showed frequent turns and in general a more complex locomotor behavior than the first run. In order to test for such changes, the values were averaged for each day and each behavioral variable (Figure 3 ).

Although part of the variables had to be averaged from day to day over different groups according to the experimental design, these curves show surprisingly different trends. Activity declined only modestly but regularly over the 12 testing days. However, significance was still achieved for the difference between the first and the last day due to the relatively small interindividual variation on this measure. A greater decline than with activity was found with intrasession activity decrease. The combined evidence of these two variables is consistent with observations made during the experiment that both the initial burst of activity and the subsequent decrease tended to become smaller in the course of repeated exposures.

A more than threefold and highly significant increase was found in the number of turns from the first to the last day. The changes of choice behavior at branchings followed a different trend. The frequency of entering radial alleys leading inward remained almost unchanged. The frequency of entering radial alleys leading outward was initially high but declined gradually to the level of the frequency for entering the inward leading alleys. The preference for outward as against inward leading alleys was therefore not constant, but tended to vanish over repeated exposures. The frequency of reentering the same hexagonal section from which the radial alley was previously entered was relatively small at the begin. ning, but increased steadily toward the end of the experiment.

Finally, the transition times can be divided into two distinct groups with respect to their development over the 12 days. The transition times at straight alley sections and corners increased slightly over the 12 days at a rate roughly comparable to the simultaneous decrease of activity. The transition times at reentry into the hexagonal alleys, on the other hand, were almost doubled during the course of the 12 testing days. 


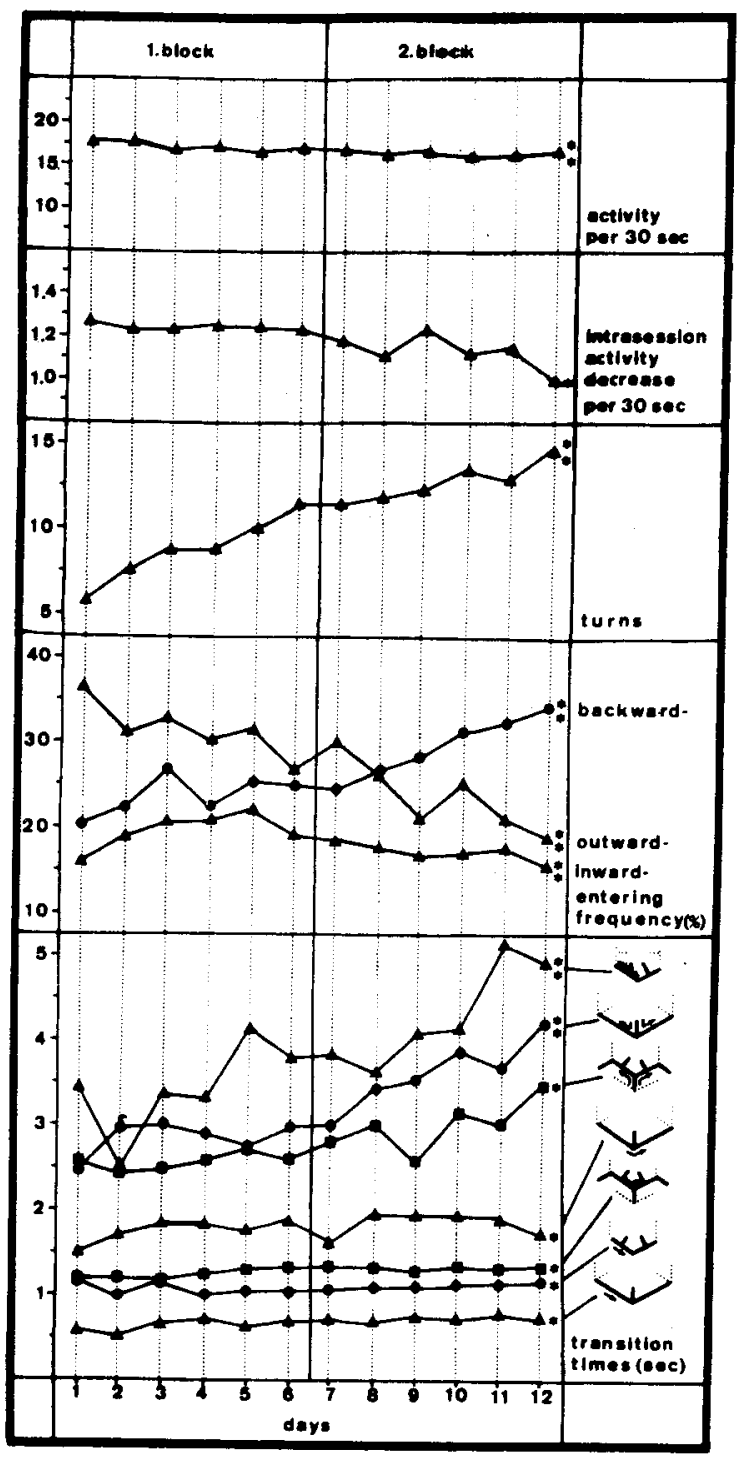

Figure 3. Daily averages of activity, intrasession activity decrease and turns combined for all six mazes. The daily averages of the remaining variables were combined for the corresponding mazes only. (Entering frequencies: mazes 3 and 6 for entering outward leading radial alleys, mazes 4,5 , and 6 for entering inward leading radial alleys, mazes $3,4,5$, and 6 for leaving radial alleys with backward reentering of hexagonal alleys. Transition times: combined for the mazes containing the distinct situations shown on the figure.)

$*=\mathrm{p}<0.05 ; * *=\mathrm{p}<0.01$.

\section{DISCUSSION}

Different aspects of maze geometry, as well as experience, had effects on locomotor behavior in this study. The positive influence on activity of increased area and the negative influence of increased maze complexity can be regarded in terms of the incentive value of the mazes. The further the "interesting" places are from each other, the more locomotion is required to reach these places. On the other hand, the more interesting places there are per unit of area (in other words, the higher the density of distinct stimuli) the less locomottion is required for the same amount of exploration. These observations might therefore be considered in the light of such studies as those of Berlyne and Slater (1957) and of Montgomery (1954), which found that rats prefer the arm of a $\mathrm{T}$ - or Y-maze leading to a complex goal. A further influence of maze geometry was seen in the tendency to prefer alleys leading toward the periphery. This particular finding is comparable with the observations of Dashiell and Bayroff (1931), Schneirla (1933), and Bättig, Zahner, and Grandjean (1964), who interpreted this in terms of "forward" or "centrifugal swing" tendencies. Finally, activity was influenced by distinct maze locations. Locomotion speed was highest in straight alley sections, decreased at corners and alley branchings, and reached the lowest levels at reentries from radial side alleys into a hexagonal alley. These differences underline the slowing effect of alley complexity on locomotion.

The effects of experience on these variables are complex. Overall activity decreased only modestly over the 12 testing days. This finding is in line with observations on activity and intrasession activity decrease obtained earlier by repeatedly presenting the same, but not intersectioned, maze (Bättig \& Wanner, 1967; Bättig, 1969). However, in the present study the relative constancy of the quantitative aspects of locomotion were paralleled by apparent qualitative changes of locomotion. The most apparent change was in the tendency toward increased locomotion complexity, as evident from the almost threefold increase in the number of turns and the increased irregularity of locomotion speed. Velocities at different maze locations were differentially influenced by experience. Movements along straight alleys, corners or radial alleys slowed moderately and were roughly proportional to the slightly decreased amount of activity. In contrast, the times for reentering into the hexagonal alley after leaving a radial alley increased considerably. In addition, the rats returned from these places with increasing frequency to the hexagonal section from which the side alley was previously entered instead of moving forward in the same direction. The weakening of this stereotyped "forward running" was accompanied by an almost complete loss of the "centrifugal swing" behavior. The initially dominant preference for outward leading alleys vanished over the testing period, while the frequencies for inward leading alleys remained unchanged. The combined evidence of increased irregularity of locomotion speed, increased number of turns, and lowered peripheral and forward preferences suggests that a general trend toward less stereotyped locomotion continued to increase in the course of exposures to the daily changing maze arrangements. This trend may be seen to reflect a shift of the behavior toward a more 
detailed investigation of "special" parts of the mazes. The balance between the two competing tendencies of exploring more sections per unit time versus more details per section as proposed by Eisenberger (1972) might therefore depend upon experience, among other possible factors. Whether this balance might further depend upon the stimulus content of an exploratory situation and its daily change or not can be answered only tentatively on the basis of the present results. The relevant comparisons between first and second presentation of the six mazes revealed the same differences in intermaze activity and intrasession activity decrease for both experimental blocks. On the other hand, the number of turns, which in this respect is of special importance, appeared to have a tendency to change from the first to the second block, the amount of change being dependent upon complexity of the mazes. Therefore the suggested conclusion that besides experience, situational complexity might also affect the balance between the two opposing tendencies, will need further experimentation. However, the major significance of these findings lies in pointing out the potential value of behavioral variables other than measures of simple activity for the detection and future analysis of the apparently complex behavioral processes involved in maze exploration.

\section{REFERENCES}

Büttig, K. Drug effects of exploration of a combined maze and open-field system by rats. Annals of the New York Academy of Sciences, 1969, 159, 880-897.
Bättig, K., \& Rhiner, A. Kontinuierliche und automatische Registrierung der spontanen Lokomotion von Ratten in einem komplexen Labyrinth. Helvetica Physiologica et Pharmacologica Acta, 1966, Acta 24, C 65-C 66.

Bättig, $K$., \& Wanner, $H$. U. Die spontane Lokomotion von Ratten in einer Kombination von Labyrinthgängen und Offenfeld. Helvetica Physiologica et Pharmacologica Acta. 1967. Acta 25, 249-261.

Bättig, K., Zahner, H., \& Grandjean, E. Untersuchungen über die spontane Alternation der Ratte. Zeitschrift fur experimentelle und angewandte Psychologie, 1964, XI, 1-26.

Berlyne, D. E., \& Slater, J. Perceptual curiosity, exploratory behavior, and maze learning. Journal of Comparative and Physiological Psychology, 1957, 50, 228-231.

Dashiell, J. F., \& Bayroff, A. G. A forward going tendency in maze running. Journal of Comparative and Physiological Psychology, 1931, 12, 77-94.

Dember, W. N \& Earl, R. W. Analysis of exploratory, manipulatory, and curiosity behaviors. Psychological Review. 1957, $64,91-96$

Dutch, J., \& Brown, L. B. Visual stimulus complexity and approach behavior in rats. Psychonomic Science, 1971, 22, $162-163$.

Eisenberger, R. Explanation of rewards that do not reduce tissue needs. Psychological Bulletin, 1972, 77, 319-339. Fowler, H. Curiosity and exploratory behavior. New York: Macmillan, 1965.

Glanzer, M. Changes and interrelations in exploratory behavior Journal of Comparative and Physiological Psychology, 1961, 54, 433-438.

Montgomery. $K$. C. A test of two explanations of spontaneous alternation. Journal of Comparative and $\mathrm{Pby}$ siological Psychology, 1952, 45, 287-293.

Montgomery, $K$. C. The role of the exploratory drive in learning. Journal of Comparative and Physiological Psychology, 1954, $47,60-64$.

Schneirla, T. C. Some comparative psychology. Journal of Comparative and Physiological Psychology, 1933, 16 , 307-315

Taylor, G. T. The incentive value of complexity. Psychonomic Science, $1971,22,143-144$.

(Received for publication May 16, 1975 Revision accepted September 10,1975.) 